\title{
V4641Sgr - A super-Eddington source enshrouded by an extended envelope
}

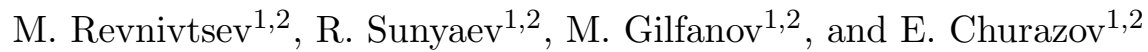 \\ 1 Max-Planck Institut für Astrophysik, Karl-Schwarzschild-Str. 1, 85748 Garching, Germany \\ 2 Space Research Institute, Russian Academy of Sciences, Profsoyuznaya 84/32, 117810 Moscow, Russia,
}

Received 18 September 2001 / Accepted 18 January 2002

\begin{abstract}
Optical spectroscopy of an unusual fast transient V4641 Sgr constrains its mass to be $8.7-11.7 M_{\odot}$ (9.6 $M_{\odot}$ is the best fit value) and the distance to $7.4-12.3 \mathrm{kpc}$ (Orosz et al. 2001). At this distance the peak flux as measured by ASM/RXTE in 2-12 keV energy band implies the X-ray luminosity exceeding $2-3 \times 10^{39} \mathrm{erg} \mathrm{s}^{-1}$, i.e. near or above the Eddington limit for a $9.6 M_{\odot}$ black hole. Optical photometry shows that at the peak of the optical outburst the visual magnitude increased by $\Delta m_{V} \gtrsim 4.7^{\mathrm{m}}$ relative to the quiescent level and reached $m_{V} \lesssim 8.8^{\mathrm{m}}$. An assumption that this optical emission is due to the irradiated surface of an accretion disk or a companion star with a black body spectrum would mean that the bolometric luminosity of the system exceeds $\gtrsim 3 \times 10^{41} \mathrm{erg} \mathrm{s}^{-1} \sim 300 L_{\text {Edd }}$.

We argue that the optical data strongly suggest the presence of an extended envelope surrounding the source which absorbs X-ray flux and reemits it in the optical and UV. The data also suggest that this envelope should be optically thin in UV, EUV and soft X-rays. The observed properties of V4641 Sgr at the peak of an optical flare are very similar to those of SS433. This envelope is likely the result of a near- or super-Eddington rate of mass accretion onto the black hole. The envelope vanishes during subsequent evolution of the source when the apparent luminosity drops well below the Eddington value. Thus this transient source provides us with direct proof of the dramatic change in the character of an accretion flow at the mass accretion rate near or above the critical Eddington value as predicted long ago by the theoretical models.
\end{abstract}

Key words. V4641

\section{Introduction}

The X-ray transient V4641 Sgr was discovered in February 1999 (in’t Zand et al. 1999; Markwardt et al. 1999a) and since then it has demonstrated moderate X-ray activity during approximately half a year at a level of $\sim 10 \mathrm{mCrab}^{1}$ (Markwardt et al. 1999b; Revnivtsev et al. 2002). In September 1999 it entered a period of violent X-ray and optical activity, during which the X-ray flux peaked at $\sim 12$ Crab (Smith et al. 1999) and observed optical brightness peaked at $m_{V} \sim 8.8$ (Stubbings 1999; Kato et al. 1999). After a few days of strong activity in the optical (Kato et al. 1999), radio (Hjellming et al. 2000) and X-ray (Revnivtsev et al. 2002) bands the source became undetectable in radio and X-rays, and returned to the quiescent level in the optical. It should be noted that unusual optical activity of this object was discovered more than 20 years

\footnotetext{
Send offprint requests to: M. Revnivtsev

1 For a spectrum as for the Crab X-ray source, $1 \mathrm{mCrab}$ translates to an energy flux of $3 \times 10^{-11} \mathrm{erg} \mathrm{s}^{-1} \mathrm{~cm}^{-2}$ in the 2 to $12 \mathrm{keV}$ bandpass
}

ago by Goranskij (1978), indicating that the source is a recurrent transient.

A detailed analysis of the $\mathrm{X}$-ray data will be reported elsewhere (Revnivtsev et al. 2002). In this article we concentrate on the implications of the available optical data (Goranskij 1978; Goranskij 1990; Kato et al. 1999; Orosz et al. 2001) and demonstrate that these data strongly suggest the presence of an extended envelope surrounding the source.

\section{Optical evidence for the envelope}

Optical spectroscopic observations of V4641 Sgr performed at quiescence led to its identification as a high mass black hole binary with an orbital period of $\approx 2.81$ days and primary and secondary masses of $\approx 9.6 M_{\odot}$ and $\approx 6.5 M_{\odot}$. Optical observations also constrain the source distance to 7.4-12.3 kpc (Orosz et al. 2001). This new distance measurement dramatically changed our understanding of the source. At this distance the peak flux of 12 Crab measured by ASM/RXTE implies that the $2-12 \mathrm{keV}$ luminosity of the source exceeds $2-3 \times 10^{39} \mathrm{erg} \mathrm{s}^{-1}$, i.e. it is near to or above the Eddington luminosity for a $9.6 M_{\odot}$ black hole. 
The most remarkable property of V4641 Sgr was revealed by optical photometry during the giant outburst in Sep. 1999. The available optical and X-ray data are shown in Fig. 1. The whole outburst was rather short and there is a gap in optical data between Sep. 14.8 and 15.4. The X-ray data are mostly those of ASM/RXTE with a sampling interval of approximately 1.5 hours. The existing optical data suggest that the optical brightness started to increase around Sep. 14.8, 1999, approximately at the time of the first outburst in X-rays. The peak of the optical brightness however does not coincide with any of the 3 peaks observed in X-rays. Moreover, during the peak of X-ray flux on $\sim$ Sep. 15.7, 1999 the optical flux shows a steady decline uncorrelated with the behavior in X-rays. In general the data shown in Fig. 1 suggest a relatively stable evolution of the optical flux and an erratic multipeaked behavior in the X-rays (the amplitude of X-ray variations is much higher that that of the optical emission). The peak in the optical flux appears to coincide with the minimum of the X-ray flux although the possibility of short outburst(s) occurring between the X-ray points can not be entirely excluded.

A lower limit on the peak source luminosity can be obtained from the ASM data. The maximum flux measured by ASM during the giant outburst was $\approx 12.1$ Crab. Since the ASM observation probably missed the peak of the outburst, the actual peak flux may be higher. Using the source count rates in three ASM energy channels and the Crab count rates in these channels as a reference for the flux estimates, we can obtain the 1.3-12 keV energy flux from V4641 Sgr at the peak of the X-ray outburst $-F_{\mathrm{x}} \gtrsim 3.8 \times 10^{-7} \mathrm{erg} \mathrm{s}^{-1} \mathrm{~cm}^{-2}$. At the distance of $9.6 \mathrm{kpc}(7.4-12.3 \mathrm{kpc}$ at the $90 \%$ confidence) it would correspond to a $1.3-12 \mathrm{keV}$ luminosity in excess of $4.2 \times 10^{39}\left(2.5-6.9 \times 10^{39}\right) \mathrm{erg} \mathrm{s}^{-1}$. On the other hand, the Eddington luminosity for a $9.6 M_{\odot}\left(8.7-11.7 M_{\odot}\right)$ object is $\sim 1.3 \times 10^{39} \mathrm{erg} \mathrm{s}^{-1}\left(1.2-1.6 \times 10^{39} \mathrm{erg} \mathrm{s}^{-1}\right)$. We therefore conclude that at the peak of the outburst of V4641 Sgr the $1.2-12 \mathrm{keV}$ luminosity alone exceeded the critical Eddington luminosity by a factor of a few: $\gtrsim 3.2 L_{\text {Edd }}$ ((1.6-5.7) $\left.L_{\text {Edd }}\right)$.

Because of the gap in the data between Sep. 14.8 and 15.3 the optical observations also probably missed the peak of the optical outburst, therefore the maximal visual brightness at the peak corresponds to $m_{V} \lesssim 8.8^{\mathrm{m}}$. The interstellar reddening in the direction of the source, $E(B-V)=0.32 \pm 0.1$ (Orosz et al. 2001), corresponds to an extinction of $A_{V} \approx 3.2 E(B-V) \approx 1.0 \pm 0.3$. The extinction corrected flux at $\lambda \sim 0.55 \mu \mathrm{m}$ exceeds $F_{\nu} \gtrsim 6.7 \times 10^{-6} \mathrm{erg} \mathrm{s}^{-1} \mathrm{~cm}^{-2} \mathrm{keV}^{-1}$, which approximately corresponds to a source luminosity in the optical spectral band of $L_{\mathrm{opt}} \sim 10^{38.2} \mathrm{ergs} \mathrm{s}^{-1}$. Note that even the optical data alone give us the near-Eddington source luminosity.

An increase of optical flux is observed during X-ray outbursts in many X-ray binaries (e.g. van Paradijs \& McClintock 1995). The star itself can not change its internal brightness significantly in a time scale of days or

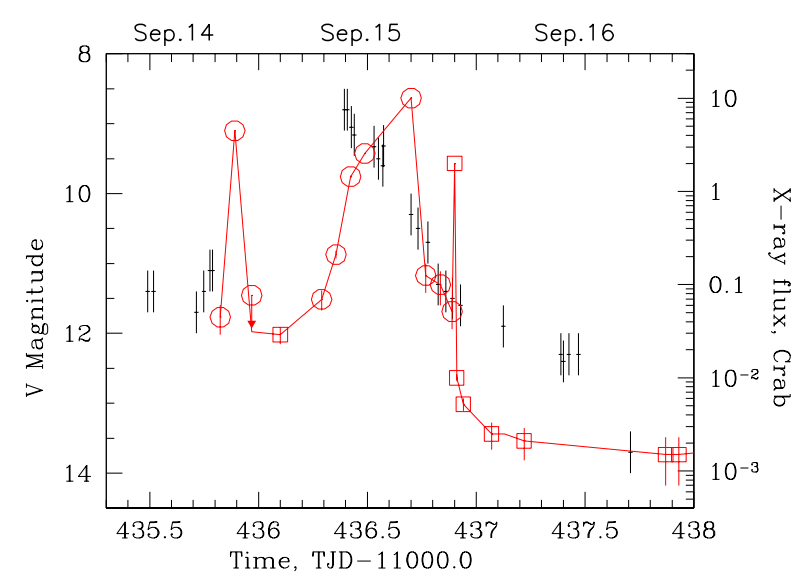

Fig. 1. The light curves of V4641 Sgr in the optical $V$-band (crosses, from Kato et al. 1999) and and in X-rays near the peak of main outburst in Sep. 1999. The RXTE/ASM points $(1.5-12 \mathrm{keV})$ are shown by open circles, the RXTE/PCA (3$20 \mathrm{keV}$ ) - by open squares (Revnivtsev et al. 2002).

months. Therefore it is usually assumed that the optical emission in low mass X-ray binaries (which have very faint optical companions) is due to the irradiated accretion disk and the irradiated side of the secondary star (e.g. Lyutyi \& Sunyaev 1976). However, in the case of high mass X-ray binaries where the companion star is relatively massive and luminous (like V4641 Sgr), it is extremely difficult to change the optical luminosity of the system by a factor of $\sim 100$. To the best of our knowledge, this is the only case where the optical brightness of a high mass system changed by such a large factor. If we assume that the peak optical flux is due to the optically thick thermal emission of the accretion disk or a companion star we can estimate the bolometric luminosity of system required to explain the observed optical flux.

The size of the accretion disk $R$ in the binary system can be assumed to be a fraction $k$ of the size of the Roche lobe of the primary, where $k$, according to different estimates, is of the order of 0.3-0.6 (see e.g. Paczynski 1977). Using this radius of the emitting region and the observed optical flux we can estimate the temperature of the emitting region assuming the simplest Planck shape of the spectrum. The obtained temperature is close to $T \sim 3 \times 10^{5} \mathrm{~K}$. Since the irradiated disk is optically thick and is emitting black body spectrum its bolometric luminosity can be estimated as $2 \pi R^{2} \sigma T^{4} \sim$ few $\cdot 10^{41} \mathrm{ergs} \mathrm{s}^{-1}$. In Fig. 2 we present the black body models of the spectra of V4641 Sgr during three stages of its flux history from the bottom to the top - the quiescent, pre-outburst and the peak optical activity. The shaded areas around the spectra show the allowed regions of spectral flux densities, taking into account the freedom in the system parameters. It is immediately seen that within the irradiation model the bolometric luminosity of V4641 Sgr at the peak of optical outburst should be of the order of $10^{41}$ $10^{43} \mathrm{ergs} \mathrm{s}^{-1}$ for any choice of the binary system parameters. The same conclusion is reached if we assume that 


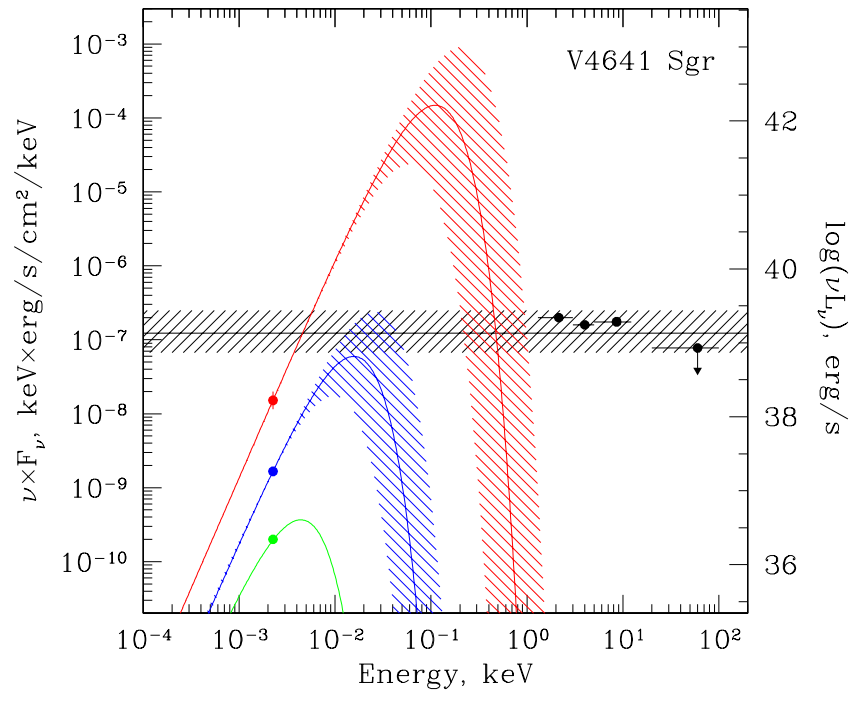

Fig. 2. The approximation of the spectra of V4641 Sgr at the different stages of the outburst by the optically thick emission model (black body) using the optical points $(\lambda \sim 0.55 \mu \mathrm{m}$, $h \nu \sim 2 \mathrm{eV})$. Upper curve corresponds to maximal observed optical brightness. In the X-ray band (1-100 keV) ASM/RXTE and BATSE/CGRO points are shown (not simultaneous with optical points). The shaded area around the curves show the allowed region, using some freedom in the system parameters. Horizontal shaded area shows the Eddington level for the black hole with mass $M_{\mathrm{BH}} \sim 10 M_{\odot}$ using different source distance in the range $7.4-12.3 \mathrm{kpc}$.

the irradiated surface of the secondary star is responsible for the observed optical flux.

It is very unlikely that a source powered by accretion can have a luminosity 2-3 orders of magnitude higher than the Eddington value. Therefore one has to consider alternative scenarios.

As was suggested already in the 1970 s (e.g. Shakura \& Sunyaev 1973), super-critical accretion onto a black hole could result in the formation of a wind, or geometrically thick envelope. Such an envelope, under certain circumstances, could be optically thick for a) X-ray photoabsorption, and b) infrared and optical emission, but still optically thin in UV, EUV and soft X-rays, where the bulk of the energy can be emitted. Below we assume that such an envelope, intercepting $\mathrm{X}$-ray flux from the source and re-radiating in optical and UV bands, is responsible for the observed optical emission. Detailed calculations of the radiation transport in the envelope is beyond the scope of our paper, but some rough estimates of the envelope temperature and density are possible.

First of all, let us assume that the optical emission has a thermal origin. If one takes a temperature of the emitting region that is too low, e.g. $T<3 \times 10^{4} \mathrm{~K}$, then given the observed optical flux of the system, the required size of the emitting region (even under the assumption of a black body spectrum) is much larger than the size of the binary system, which is unlikely. On the other hand, for a temperature of the envelope that is too high, e.g. $T>3 \times 10^{6} \mathrm{~K}$, the required size of the emitting region is smaller, but the bolometric luminosity inevitably will exceed the Eddington value even under the assumption of an optically thin emission spectrum. The size of the emitting region can only be made comparable to the binary system size with the bolometric source luminosity below the Eddington value for some intermediate values of temperature. In the discussion below we will assume a temperature of the envelope of $T \sim 3 \times 10^{5} \mathrm{~K}$. The envelope is then optically thick in the optical, is getting optically thin in UV and EUV and is optically thick for $\mathrm{X}$-ray photoabsorption.

In order to absorb (via photoabsorption) most of the energy emitted in X-rays the hydrogen column density of the envelope $\int n \mathrm{~d} l$ (where $n$ is the hydrogen density of the envelope) has to be at least of the order of $10^{24-25} \mathrm{~cm}^{-2}$, which means Compton thick envelope. On the other hand, maximum radiation efficiency in the optical band is achieved if the line of sight emission measure of the envelope is $\int n^{2} \mathrm{~d} l \geq 10^{37-38} \mathrm{~cm}^{-5}$ (depending on the contribution of the opacity in the lines to the total opacity of the envelope). Such a line-of-sight emission measure implies that the envelope is optically thick in the optical band (for the assumed temperature of $\left.T \sim 3 \times 10^{5} \mathrm{~K}\right)$. Line-of-sight emission measure however should not be much larger than this value. Otherwise the envelope would become optically thick in UV and EUV and the luminosity would exceed the Eddington value. Thus, assuming a homogeneous envelope (which is obviously a large oversimplification) we can conclude that for a size of the envelope $R \sim$ few $R_{\odot}$ (i.e. of the order of size of the Roche lobe of the primary) the density should be about $n \sim 10^{12-14} \mathrm{~cm}^{-3}$. The total mass of the envelope is then $M_{\mathrm{env}} \sim \int n \mathrm{~d} V \sim 10^{23-25} \mathrm{~g}$. It is interesting that for an Eddington mass accretion rate of $\dot{M}_{\text {Edd }} \sim 10^{19} \mathrm{~g} \mathrm{~s}^{-1}$ (for a $M \sim 10 M_{\odot}$ black hole) such a mass can be accumulated during $10^{4}-10^{6} \mathrm{~s}$. The cooling time of the envelope is much shorter - of the order of seconds.

Thus the optical data suggest that there is an absorbing/reprocessing envelope surrounding the source. In such a model a relatively smooth behavior of the optical luminosity would reflect the total intrinsic luminosity of the source and the amount of $\mathrm{X}$-rays absorbed by the reprocessing region. The evolution of $L_{\mathrm{opt}} / L_{\mathrm{x}}$ suggests that the geometry of absorbing/reprocessing region changes with time. At the maximum of the optical light curve most of the X-rays are probably absorbed, while later (at the $\mathrm{X}$-ray maximum) the dominant fraction of $\mathrm{X}$-rays is directly observed. Rapid changes in $\mathrm{X}$-ray flux could be due to changes of the geometry of the absorbing region (e.g. edge of the torus obscuring the line of sight) or thermal instability in the gas which causes fragmentation of the medium into separate clouds. During these transitions the distribution of the energy emitted in different energy bands (e.g. in optical/UV and in X-rays) could change strongly depending on the parameters of the envelope, while a bolometric luminosity of the source behaves much more smoothly and reflects the mass accretion rate onto the black hole. 
Note that the enhanced optical activity, observed during approximately 2 weeks before the giant outburst (the middle curve in Fig. 2 of Kato et al. 1999) and the optical activity observed by Goranskij (1978) could also be explained in the frame of a model with an emitting extended envelope. During these observations $m_{V}$ was $\sim 12^{\mathrm{m}}$ (two magnitudes brighter than during quiescence). Assuming a black body shape of the spectrum and a size of the emitting region of the order of the size of the accretion disk one can estimate the temperature of the emitting region to be of the order of $40000-50000 \mathrm{~K}$. This temperature is again far too high for the irradiated disk or a star (e.g. Lyutyi \& Sunyaev 1976), especially taking into account the low X-ray luminosity of the source, observed simultaneously (Revnivtsev et al. 2002). The observed optical flux at this stage could still correspond to near-Eddington bolometric luminosity if the temperature of the envelope is 10 times lower than what it is during the brightest optical flare. This is possible if the line-of-sight emission measure and the geometrical dimensions of the envelope have not changed. The optical depth for UV and EUV radiation in this case would be much higher.

As the mass accretion rate onto the black hole drops below the Eddington value the envelope vanishes. Indeed, the available X-ray and optical observations of the source before and after Sep. 1999 (see Revnivtsev et al. 2002) do not provide any evidence that the source at that time was different from other X-ray transients. We have not found any evidence of super-Eddington accretion or an envelope. Therefore we believe that the envelope forms only during the episodes of near- or super-critical accretion rate, and disappear at lower accretion rates.

Depending on the envelope outflow velocity it can either contract to an accretion disk, or leave the binary system. In the latter case it can be detected. In the absence of external heating the cooling time of such an envelope is small, $\sim 10-100 \mathrm{~s}$. Therefore, in such a simple picture it could be detected through an increased $N_{\mathrm{H}} L$ value. Assuming that during the expansion the density of the envelope changes as $n \propto R^{-2}$ and the expansion velocity is of the order of the orbital velocity $\left(v \sim 300 \mathrm{~km} \mathrm{~s}^{-1}\right)$, one can expect $N_{\mathrm{H}} L \sim 10^{22-23}(\Delta t / \text { days })^{-1}\left(v / 300 \mathrm{~km} \mathrm{~s}^{-1}\right) \mathrm{cm}^{-2}$. This extra absorption would become comparable with the interstellar value of $N_{\mathrm{H}} L \sim 3 \times 10^{21} \mathrm{~cm}^{-2}$ on a time scale of a week.

We would like to emphasize, that the observations of outburst of V4641 Sgr in 1999 strongly suffered from the absence of broad band photometry and precise optical, infrared and UV spectroscopy. During the next outburst of the source such data would be extremely helpful for investigation of the envelope.

What triggered the major outburst of V4641 Sgr is not clear. Two standard models could be mentioned - the mass transfer instability and the disk transfer instability (see e.g. Lasota 1996 for a review). The mass transfer instability associated with the internal structure changes in the secondary star has a typical time scale much longer than days and therefore can not explain the observed short outburst. External heating of the secondary star by X-rays may help, but prior to the outburst the X-ray flux of the source was approximately stable over a period of $\sim 6$ months. On the other hand, the disk transfer instability model (in which partial ionization of hydrogen plays a role) was successful in explaining the light curves of X-ray transients (e.g. Meyer 1982) and can provide the required time scales. Note, however, that the proximity of the hot companion star with a surface temperature of $T \sim 10000 \mathrm{~K}$ may significantly alter the ionization structure of the accretion disk and the behavior of the instability.

\section{Comparison with SS 433}

The difficulties with the estimates of the bolometric luminosity of V4641 Sgr during the outburst described above resemble those with the well known source SS 433. Hubble Space Telescope observations of SS 433 have detected the source in UV at $\sim 1500 \AA$. The brightness temperature of the UV emission was estimated to be $T \sim 72000 \mathrm{~K}$ (Dolan et al. 1997). The energy flux, observed in UV spectral band is $\sim 2 \times 10^{-16} \mathrm{ergs} \mathrm{s}^{-1} \mathrm{~cm}^{2} \AA^{-1}$. After the correction for the interstellar absorption, the luminosity of the source in UV is $L \sim 10^{39} \mathrm{ergs} \mathrm{s}^{-1}$. The bolometric source luminosity, calculated using the parameters of the opticalUV emission, presented in Dolan et al. (1997) is around $L_{\mathrm{bol}} \sim 1.3 \times 10^{40} \mathrm{ergs} \mathrm{s}^{-1}$. This value is strongly superEddington even for a compact object with a mass $10 M_{\odot}$. However, if we assume that in the case of SS 433 we also observe the envelope, which is optically thick in the optical and in the UV spectral bands, but still optically thin in EUV spectral band, then the estimate of the source bolometric luminosity can be strongly reduced, and become close to Eddington luminosity for the $10 M_{\odot}$ black hole.

\section{Conclusion}

In this article we showed that the optical observations of X-ray transient V4641 Sgr during the powerful outburst in Sep. 14-15, 1999 strongly suggest that there was a massive envelope enshrouding the source. This envelope was probably the result of a near or super Eddington rate of mass accretion onto the black hole during this period. At that time the V4641 Sgr closely resembled another unique object - SS 433, which is also thought to be a super Eddington accretor. The fraction of bolometric luminosity emitted in different energy bands strongly depends on the parameters of the envelope and, thus, the observed optical and X-ray fluxes can evolve in a complicated and uncorrelated way.

Thus this transient source provides us the first direct proof of the dramatic change in the character of an accretion flow at a mass accretion rate near or above the critical Eddington value as was predicted long ago by the theoretical models. One can propose that the observed pattern of optical/X-ray long term variability observed for this 
source could be an indicator of a super-Eddington accretion in the binary system. However, it is still not very clear what was the reason for the violent behavior of V4641 Sgr.

Acknowledgements. Authors thank an anonymous referee for helpful comments. This research has made use of data obtained through the High Energy Astrophysics Science Archive Research Center Online Service, provided by the NASA/Goddard Space Flight Center. The work was done in the context of the research network "Accretion onto black holes, compact objects and protostars" (TMR Grant ERB-FMRX-CT98-0195 of the European Commission).

\section{References}

Dolan, J., Boyd, P., Fabrika, S., et al. 1997, A\&A, 327, 648

Goranskij, V. P. 1978, Astrn. Tsirk., 1024, 3

Goranskij, V. P. 1990, Inf. Bull. Variable Stars, 3464

Hjellming, R., Rupen, M., Hunstead, R., et al. 2000, ApJ, 544, 977

in't Zand, J., Heise, J., Bazzane, A., et al. 1999, IAUC, 7254 in't Zand, J., Kuulkers, E., Bazzano, A., et al. 2000, A\&A, 357, 520

Kato, T., Uemura, M., Stubbings, R., et al. 1999, Inf. Bull. Variable Stars, 4777

Lasota, J.-P. 1996, Compact stars in binaries, Proceedings from IAU 165, ed. J. van Paradijs, E. Peter, J. van den Heuvel, \& E. Kuulkers (Kluwer Academic Publishers, Dordrecht), 43

Lyutyi, V., \& Sunyaev, R. 1976, Sov. Astron., 20, 290

Markwardt, C., Swank, J., \& Marshall, F. 1999a, IAUC, 7120

Markwardt, C., Swank, J., \& Morgan, E. 1999b, IAUC, 7257

Meyer, F. 1982, in Accreting Neutron Stars, 333, SEE N83-15179 05-88

Orosz, J., Kuulkers, E., van der Klis, M., et al. 2001, ApJ, 555, 489

Paczynski, B. 1977, ApJ, 216, 822

Revnivtsev, M., Gilfanov, M., Churazov, E., \& Sunyaev, R. 2002, A\&A, submitted

Smith, D., Levine, A., \& Morgan, E. 1999, IAUC, 7253

Shakura, N. I., \& Sunyaev, R. A. 1973, A\&A, 24, 337

Stubbings, R. 1999, IAUC, 7253

van Paradijs, J., \& McClintock, J. 1995, in X-ray Binaries, ed. W. H. G. Lewin, J. van Paradijs, \& E. P. J. van den Heuvel (Cambridge: Cambridge Univ. Press), 58 